\title{
Steel Tape-Wound Cut Cores as Magnet Yokes for the Beam-Dump Kickers of the Large Hadron Collider
}

\author{
Manfred Mayer, David Fox, Francesco Castronuovo, and Urban Jansson
}

\begin{abstract}
Fast-pulsed magnets, also called kickers, are used in particle accelerators for beam injection, extraction and similar applications. To excite these magnets, typically current pulses with rise and fall times in the range of $100 \mathrm{~ns}$ to $10 \mu$ s are used, with a pulse duration of up to $100 \mu$ s and amplitudes in the order of kilo amperes. The short rise time imposes low-inductance circuits and high-voltage operation. The yokes are usually made out of ferrite, with field saturation being reached at about $0.5 \mathrm{~T}$. To remove the 7-TeV proton beams safely from the large hadron collider (LHC), 15 beam-dump kickers are used for each of the two rings which must provide an overall bending strength of $6.2 \mathrm{~T} \cdot \mathrm{m}$ with a rise time of about $3 \mu$ s and a flat top duration of $90 \mu \mathrm{s}$. For the first time steel with saturation above $1.5 \mathrm{~T}$ has been used as yoke material for such a short rise time. Due to eddy current losses the yokes must be laminated. The lamination thickness of $50 \mu \mathrm{m}$ is determined by the frequency spectrum of the current pulse and by the steel quality. The insulation layer of the laminations shall withstand a voltage of $1 \mathrm{~V} /$ layer, which is difficult to achieve at the cut edges. As stamping, handling and assembly of 1.2-m-long magnets with such thin sheets would be extremely difficult, the concept of tape-wound $\mathrm{C}$-shaped cut cores was developed. Two cores are moulded together in charged epoxy resin with a thin intermediate insulator. The yoke is finally assembled from a series of such "twin packs," using threaded inserts for precision mounting. This paper discusses the choice of the steel quality and lamination thickness, and addresses technical challenges related to the manufacturing of the $\mathrm{C}$-cores, arising from the tight mechanical, electrical and magnetic requirements.
\end{abstract}

Index Terms-HF transformers, magnetic cores, transformer cores.

\section{INTRODUCTION}

\section{A. Introduction to CERN and the LHC Project}

$\mathbf{T}$ $\checkmark$ he European Organization for Nuclear Research (CERN) is an intergovernmental organization with 20 member states. It has its seat in Geneva. Its objective is to provide for collaboration among european states in the field of high energy particle physics research. At present, more than 5000 physicists from research institutes world-wide use the CERN installations for their experiments.

Manuscript received October 15, 2003.

M. Mayer is with Division EST, European Organization for Nuclear Research (CERN), CH 1211 Geneva, Switzerland (e-mail: manfred.mayer@cern.ch).

D. Fox is with Cogent Power Limited, Newport/South Wales NP19 OXT, U.K. (e-mail: david.fox@ cogent-power.com).

F. Castronuovo and U. Jansson are with Division AB, European Organization for Nuclear Research (CERN), CH 1211 Geneva, Switzerland (e-mail francesco.castronuovo@cern.ch; urban.jansson@cern.ch).

Digital Object Identifier 10.1109/TMAG.2004.829189

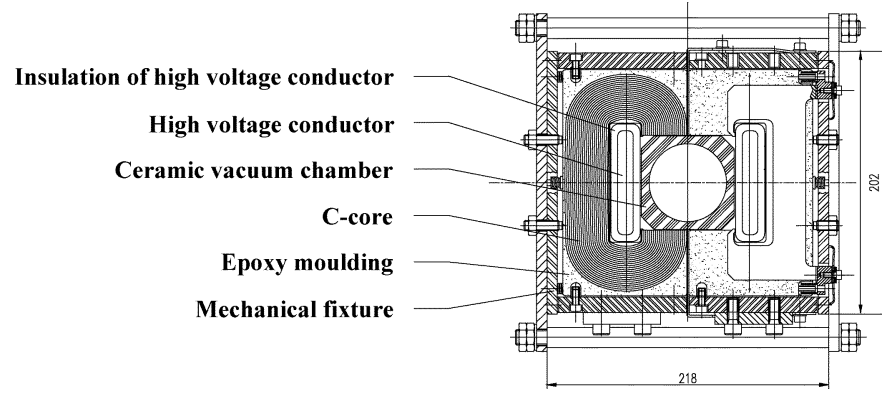

Fig. 1. Cross-section of an extraction kicker magnet.

The large hadron collider (LHC) is the accelerator under construction on the CERN site [1]. The LHC machine will mainly accelerate and collide $7-\mathrm{TeV}$ proton beams as well as heavier ions such as lead. It will be installed in the existing 27-km circumference tunnel, about 100-m underground, previously housing the large electron positron collider (LEP). The LHC is scheduled to come into operation in the year 2007. Two extraction systems are required for beam dumping in order to remove the counter-rotating beams safely from the collider during setting up of the accelerator, at the end of a physics run and in case of emergencies. Each system consists of 15 extraction kickers and 10 dilution kickers, six vertical (V) and four horizontal $(\mathrm{H})$.

\section{B. Introduction to Fast-Pulsed Magnets}

A typical cross-section of a fast-pulsed magnet is shown in Fig. 1. The yoke is composed of tape-wound cores of thin Si-steel, into which a gap is cut. Depending on the magnet type, either two or three C-cores are moulded into a unit. Several of these units assembled in a mechanical fixture form the magnet yoke. The magnet is powered by an excitation coil. The magnet and its excitation coil can be opened horizontally in order to insert a ceramic vacuum chamber, with its two large vacuum flanges reaching out at both ends. A pulse generator energizes each magnet. For the extraction kickers the maximum voltage over the magnet is $30 \mathrm{kV}$. The magnet current pulse has an amplitude of $20 \mathrm{kA}$ with a rise time of $2.8 \mu \mathrm{s}$ and a flat top duration of $90 \mu$ s followed by an approximately exponential decay of $1900 \mu \mathrm{s}$. This current induces a magnetic field in the steel of $0.6 \mathrm{~T}$ assuming a stacking factor of 0.88. The initial part of the magnet flux pulse is shown in Fig. 2. The required magnetic field for the dilution kickers are $0.85 \mathrm{~T}(\mathrm{H})$ and $0.89 \mathrm{~T}(\mathrm{~V})$, respectively. These magnetic fields cannot be achieved with ferrites which saturate at $0.5 \mathrm{~T}$ 


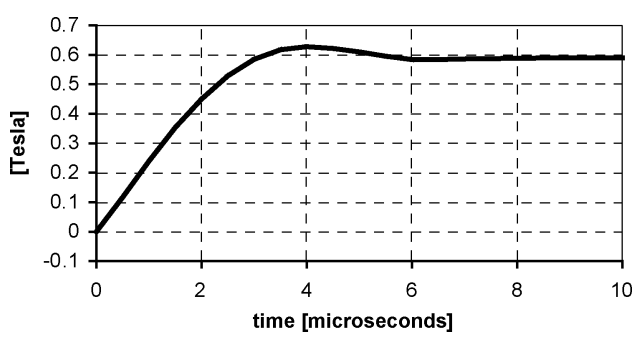

Fig. 2. Magnetic flux in the core of an excitation kicker magnet.

\section{TAPE-WOUND CORES}

\section{A. General}

During several years the magnet cores for the LHC beam-dump kickers have been the subject of intense R\&D efforts. CERN has collaborated closely with industry to find a replacement for ferrites, which are normally used as magnet yokes in fast-pulsed magnet applications. Given the relatively slow rise time, the possibility to use laminated steel yokes were investigated. As stamping, handling, and assembly of 1.2-m long magnets with such thin sheets would be extremely difficult, the concept of tape-wound $\mathrm{C}$-shaped cut cores was developed.

\section{B. Radiation Resistance}

The service life of the moulded C-core assemblies is expected to at least 20 years and during this period they will be exposed to an integrated radiation dose of at least $10^{6} \mathrm{~Gy}\left(10^{8} \mathrm{rad}\right)$. Therefore, only material complying with this requirement can be selected.

\section{Steel Quality and Surface Insulation}

Several factors had to be taken into account when selecting the lamination material to be used, these include:

- high saturation induction;

- gauge;

- dimension consistency;

- permeability at $150 \mathrm{kHz}$;

- ease of machining;

- high insulation resistance;

- and ease of acid etching-i.e., high iron content.

Grain oriented silicon steel with $50 \mu \mathrm{m}$, was chosen for its high saturation induction and ease of machining/acid etching (manufactured by Arnold Engineering). Thin gauge electrical steel was developed in the late 1940s / early 1950s. The original impetus for its development was the need for an improved material to be used in radar transmitting equipment and special transformers. One of the most important applications continues to be high-power pulse transformer. C-cores made from 25 and $50 \mu \mathrm{m}$ strips are especially well suited for pulses of $0.25-3 \mu \mathrm{s}$ duration.

An important consideration is also the interlaminate resistance of the core. The insulated coating must be able to withstand the temperature cycle for the stress relieving operation and the resistance must still be high enough to prevent breakdown due to the high induced voltages. The insulation coating used in this application is a modified AISI-C5 type coating. This type of coating is classified as an inorganic insulation with ceramic fillers added to enhance the surface resistance to about $5 \Omega$.

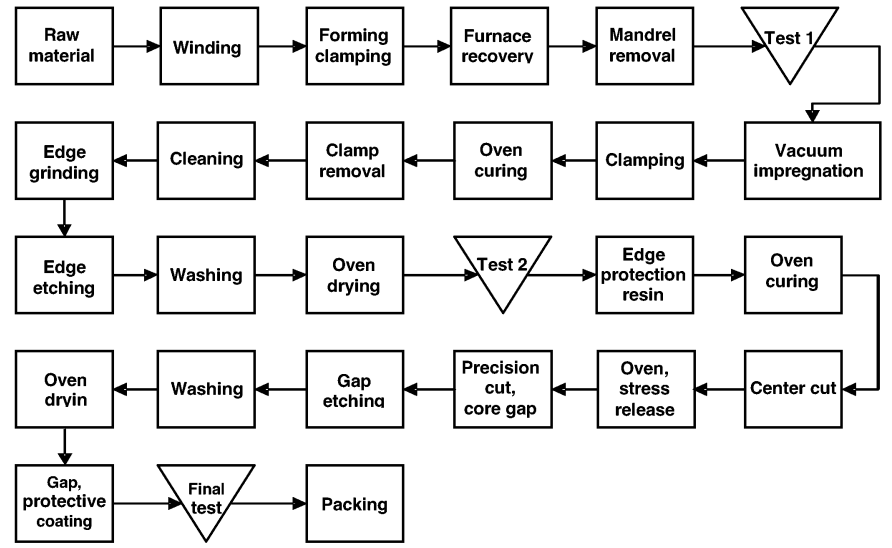

Fig. 3. C-core production route.

\section{C-Core Manufacturing}

The manufacture of an industry standard C-core has five main production steps: winding, heat treatment, impregnation, cutting, and test. However, due to the tight mechanical tolerances and magnetic requirements necessary for this application, several additional operations and control stages are needed. Fig. 3 shows a basic block diagram of the production route.

1) Winding-The core is wound with constant tension and with the edges square and parallel to within a few tens of a millimeter. Very tight tolerances on gauge, strip-width, burr height, edge bow, and ripple were demanded of the raw material supplier.

2) Forming \& Clamping-after winding the cores are clamped in special frames to ensure that the dimensions are maintained within $0.1 \mathrm{~mm}$ during heat treatment.

3) Heat treatment-in controlled nitrogen atmosphere.

4) Intermediate test—core losses and magnet current are measured.

5) Impregnation - carried out under vacuum with a radiation resistant epoxy resin.

6) Clamping - with additionally PTFE shims, between the cores and the frames to prevent bonding together during curing.

7) Curing - to reduce polymerization-bonding stresses.

8) Edge grinding - both sides of the cores are ground. After this process all laminations are short circuited.

9) Etch and resin protection coating - nitric acid solution is brushed on the ground surfaces to "burn off" the burrs.

10) Center cut and heat stabilization-first a small gap is cut in the center of the gap position. The core is then heat stabilized. This causes slight deformation of the core.

11) Gap cut-the final precision gap is cut on a machine, which provides high quality surface finish.

12) Trim/size - laminations are now peeled off, to ensure the final dimensions of the C-core.

13) Etch gap faces - to remove burrs created by the gap cutting the gap surface is etched, washed, dried, and protected.

14) Voltage holding and resistance test-as described in Section II-E. 


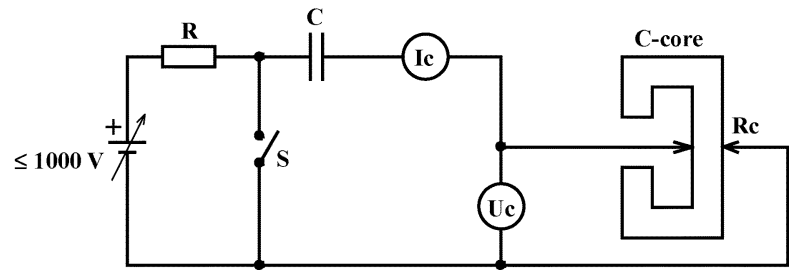

Fig. 4. Schematic test circuit.

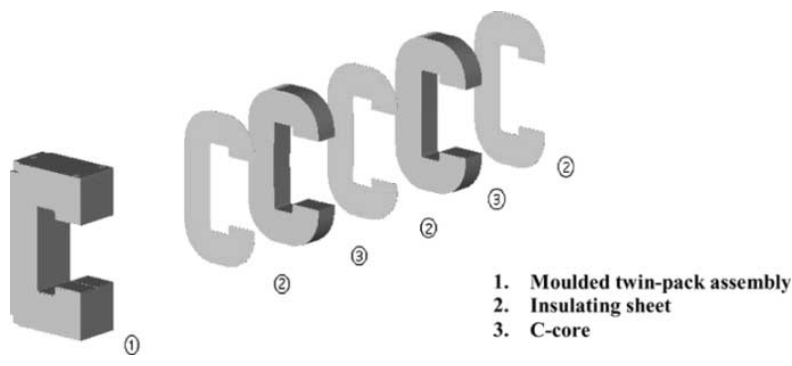

Fig. 5. Twin pack and its components.

\section{E. Core Resistance}

The voltage holding between adjacent tape layers in a C-core shall exceed $1.0 \mathrm{~V}$. To be able to verify this requirement a purposely designed test unit, as shown in Fig. 4, has been developed. It is based on the discharge of a capacitor into to a resistor via a switch.

The charging voltage and pulse duration shall correspond to $1.0 \mathrm{~V}$ per tape layer, $(600 \mathrm{~V})$ and $6 \mu$ s for the C-cores of the extraction kickers. Current and voltage waveforms are recorded. In order to obtain stable electric values, the C-cores are conditioned through 300 discharges. For the measurement, further 100 pulses are applied. The maximum required resistance $R C$ for an extraction $\mathrm{C}$-core is $20 \Omega$ and the variation between the first measurement $R C 1$, and the last measurement $R C 100$, shall remain within $\pm 10 \%$.

\section{F. Epoxy Moulding}

To provide mechanical stability and to allow positioning of the magnet gap within $\pm 0.2 \mathrm{~mm}$ precision, two or three C-cores with insulating sheets are vacuum moulded in epoxy, thus forming a twin or a triple pack assembly. The number of $\mathrm{C}$-cores per pack is determined by the operational voltage of the magnet and the voltage holding between individual layers of a C-core. To avoid flashovers between $\mathrm{C}$-cores insulating sheets are introduced. The moulding includes inserts for the fixation of the twin/triple packs. A dolomite charged epoxy compound based on the Vantico ${ }^{1}$ type F-system is used [2]. A moulded twin pack C-core assembly and an exploded view of its components is shown in Fig. 5. The dimensions of a twin

\footnotetext{
${ }^{1}$ Vantico is a registered trademark of Vantico AG, Basel, Switzerland.
}

pack for the extraction magnets are $52 \times 96 \times 176 \mathrm{~mm}$ and its weight is $3.7 \mathrm{~kg}$.

\section{G. Quality Assurance}

The quality assurance applied for the manufacturing of the moulded C-core assemblies follow strictly the LHC quality assurance plan [3]. Full traceability makes it possible to track the origin of any moulded unit back to the individually tested steel batches. Procedures for the tracking nonconformity of units are established and documented. All test records are contained in a protected electronic file. Downloading and updating via the Web is implemented.

\section{Production Status}

A prototype magnet, with slightly smaller aperture of the C-cores than the final version, has been built and extensively tested to full satisfaction. So far about 4500 of the required $5830 \mathrm{C}$-cores have been produced, continuously maintaining a high quality level. The epoxy moulding of the twin/triple packs did show some initial teething problems but is now running smoothly. A short magnet has been built with twin packs taken from the series production. It was connected in series with the prototype magnet and tested with the intended operational parameters. The measured magnetic field conforms the computer simulations made during the development phase. An acceptable phase shift of $40 \mathrm{~ns}$ between the current and the field pulse, due to the eddy current losses in the steel and the insulation resistance of the steel surfaces, has been observed.

\section{CONCLUSION}

An alternative yoke design, where thin gauge $\mathrm{Si}$-steel replaces the typically used ferrites, has been developed for fast-pulsed magnets. The industry has risen to the challenge to produce the moulded C-cores in large numbers, meeting and maintaining throughout the series manufacturing CERN's stringent requirements [4].

\section{ACKNOWLEDGMENT}

The authors wish to thank T. Slattery and T. Bui of Arnold Magnetics for their contribution in providing information on the raw material used for the C-cores. They are also in indebted to C. Serre, KA at CERN, for the design work of the magnet and its cores and to J. L. Bretin and S. Long, CERN, for the development of the $\mathrm{C}$-core tester.

\section{REFERENCES}

[1] LHC Design Report, Vol. 1, Ch. 17, CERN, 2004

[2] http://urban-jansson.web.cern.ch/Urban-Jansson/Documents/ArF_HY905_DY040_061E.pdf [Online]

[3] http://lhc-proj-qawg.web.cern.ch/lhc-proj-qawg/LHCQAP/[Online]

[4] http://urban-jansson.web.cern.ch/Urban-Jansson/Documents/IT2674\%20Spec\%20RevA.pdf [Online] 